\title{
Etude d'un terme d'erreur lié à la fonction totient de Jordan
}

par

\section{Christian Tudesq (Périgueux)}

\section{Introduction}

1.1. Historique - Notations. Si $k \in \mathbb{N}^{*}$, la fonction totient de Jordan $J_{k}$ compte, pour $n \in \mathbb{N}^{*}$, le nombre d'entiers $m, 1 \leq m \leq n$, qui ne sont divisibles par aucune des puissances $k$-ièmes des facteurs premiers de $n$. Ainsi $J_{1}$ est la fonction $\varphi$ d'Euler,

$$
J_{1}(n)=n \prod_{p \mid n}\left(1-\frac{1}{p}\right) \quad \text { pour tout } n \in \mathbb{N}^{*} .
$$

Cette relation se généralise en

(2) $\quad J_{k}(n)=n^{k} \prod_{p \mid n}\left(1-\frac{1}{p^{k}}\right) \quad$ pour tout $k \geq 1$ entier et tout $n \in \mathbb{N}^{*}$

et l'on a

$$
\sum_{n \leq x} J_{k}(n) \sim \frac{x^{k+1}}{(k+1) \zeta(k+1)} \quad(x \rightarrow+\infty) .
$$

Soit, pour tout $x \in \mathbb{R}^{+}$et tout entier $k \geq 1$,

$$
E_{k}(x):=\sum_{n \leq x} J_{k}(n)-\frac{x^{k+1}}{(k+1) \zeta(k+1)} .
$$

A la fin du dix-neuvième siècle, Sylvester a laissé entendre qu'il croyait l'inégalité $E_{1}(n)>0$ réalisée pour tout entier $n>0$. Il est amusant de noter que le contre exemple, $n=820$, fourni par Sarma en 1931, est contenu dans une table publiée par Sylvester (cf. [5]).

En 1950 Erdős et Shapiro ont prouvé

ThÉorÈme (Erdős et Shapiro [2]).

$$
E_{1}(n)=\Omega_{ \pm}\left(n \log _{4} n\right) .
$$


A ce jour le meilleur résultat sur ce sujet est dû à H. L. Montgomery:

ThÉorÈme (H. L. Montgomery [3]).

$$
E_{1}(n)=\Omega_{ \pm}\left(n \sqrt{\log _{2} n}\right) .
$$

Corollaire immédiat de (5) ou (6) : $E_{1}(n)$ change de signe une infinité de fois.

Pour $k$ entier $\geq 2$, S. D. Adhikari et A. Sankaranarayanan ont obtenu en 1990 dans [1] :

THÉORÈME A.

$$
\sum_{n \leq x} E_{k}(n) \sim \frac{x^{k+1}}{2(k+1) \zeta(k+1)} .
$$

Corollaire.

$$
\limsup _{n \in \mathbb{N}, n \rightarrow+\infty} \frac{E_{k}(n)}{n^{k}} \geq \frac{1}{2 \zeta(k+1)}
$$

THÉORÈME B.

$$
\liminf _{x \rightarrow+\infty} \frac{E_{k}(x)}{x^{k}} \leq-\frac{1}{2 \zeta(k+1)} \quad(x \text { réel }) .
$$

ThÉORÈME C. Il existe un entier strictement positif $n_{k}$ tel que

$$
n \geq n_{k} \Rightarrow E_{k}(n)>0 \quad(k \in \mathbb{N}, k \geq 2, n \in \mathbb{N}) .
$$

THÉORÈME D.

$$
\limsup _{x \rightarrow+\infty} \frac{E_{k}(x)}{x^{k}} \leq \frac{D}{\zeta(k+1)} \quad(x \text { réel }),
$$

où $D=0.7159$ pour $k=2, D=0.6063$ pour $k \geq 3$.

Les démonstrations s'appuient sur la formule de convolution

$$
J_{k}(n)=\sum_{d \mid n} \mu(d)\left(\frac{n}{d}\right)^{k},
$$

où $\mu$ est la fonction de Möbius, et sur la représentation suivante :

$$
\begin{aligned}
E_{k}(x)=x^{k} \sum_{d=1}^{+\infty} \frac{\mu(d)}{d^{k}}\left(\frac{1}{2}-\left\{\frac{x}{d}\right\}\right) & +o\left(x^{k}\right), \\
& k \in \mathbb{N}, k \geq 2, x \rightarrow+\infty, x \in \mathbb{R}^{+},
\end{aligned}
$$

où $\{u\}$ désigne la partie fractionnaire du nombre réel $u$. Soient, pour $k$ entier $\geq 2$,

$$
s_{k}:=\limsup _{x \rightarrow+\infty} E_{k}(x) x^{-k}
$$




$$
\begin{aligned}
i_{k} & :=\liminf _{x \rightarrow+\infty} E_{k}(x) x^{-k}, \\
I_{k} & :=\liminf _{n \in \mathbb{N}, n \rightarrow+\infty} E_{k}(n) n^{-k} .
\end{aligned}
$$

Adhikari et Sankaranarayanan ont aussi montré :

$$
s_{k}=\limsup _{n \in \mathbb{N}, n \rightarrow+\infty} E_{k}(n) n^{-k}
$$

et

$$
i_{k}=I_{k}-\frac{1}{\zeta(k+1)} .
$$

Le lecteur aura noté, à l'aide de (15), (16) et (18), la compatibilité des théorèmes $\mathrm{B}$ et $\mathrm{C}$.

Le lien entre $I_{k}$ et $s_{k}$ a été fait par Y.-F. S. Pétermann, dans [4] :

ThÉorÈme (Y.-F. S. Pétermann, 1991). Pour $k$ entier $\geq 2$,

$$
s_{k}+I_{k}=\frac{1}{\zeta(k+1)} .
$$

Ce dernier a également signalé, dans une communication privée a $\mathrm{M}$. Balazard, que la suite $\left(I_{k}\right)_{k \geq 2}$ est strictemement croissante.

Pour $k$ réel $\geq 1$ et $k \notin \mathbb{N}$, nous étendons la définition de $J_{k}$ par (2). L'extension des définitions de $E_{k}, s_{k}, I_{k}, i_{k}$ à des valeurs réelles strictement plus grandes que 1 de $k$, celle de (17), (18), (19), à ce même intervalle, ne soulève pas de difficultés : les démonstrations originelles s'appliquent. Comme le laissaient prévoir (5) ou (6), il n'en va pas ainsi du théorème $\mathrm{C}$.

1.2. Ennoncé des résultats obtenus. Le premier travail que nous avons mené a été d'étendre le domaine de validité du théorème $\mathrm{C}$, tout en précisant la valeur de $n_{k}$.

ThÉORÈme 1. Pour tout $n \geq 1$ entier et tout $k$ réel $\geq 2$,

$$
E_{k}(n)>0 .
$$

Nous avons également obtenu :

ThÉORÈMe 2 . Pour tout $n \geq 1$ entier et tout $k$ réel $\geq 2$,

$$
E_{k}\left(n^{-}\right)<0 .
$$

Ces résultats ne se prolongent pas au voisinage (à droite) de 1 . Nous mettons d'abord ce fait en évidence à l'aide des $\Omega$-estimations de $E_{1},(5)$ ou (6) :

\section{Proposition.}

$$
\lim _{\substack{k \rightarrow 1 \\ k>1}} s_{k}=+\infty
$$


Puis, en nous inspirant des travaux de H. L. Montgomery, nous avons obtenu une minoration de $s_{k}$ :

ThÉORÈme 3.

$$
s_{k} \geq c \sqrt{\zeta(k)} \quad(k \text { réel }>1)
$$

avec

$$
c:=\frac{1}{5 \zeta(2)} \exp \left(-\frac{1}{2} \sum_{p \geq 3} \frac{1}{p^{2}}-\frac{1}{4} \sum_{p \equiv 3(\bmod 4)} \frac{1}{p(p-1)}\right)>0.1 .
$$

Nous terminons cette étude en précisant les variations de $I_{k}$ sur un domaine non borné :

THÉORÈME 4 . Il existe un réel $k_{0}, 1<k_{0} \leq 3$, tel que $I_{k}$ soit strictement croissante sur $\left[k_{0},+\infty[\right.$.

Nous montrons que l'on peut prendre $k_{0}=1.97$ et nous conjecturons que $I_{k}$ est strictement croissante sur $[1,+\infty[$.

Tous ces résultats seront établis par des méthodes élémentaires.

\section{Inégalités concernant la fonction totient de Jordan}

2.1. Principe de la démonstration du théorème 1. On commence par donner, pour $x \in \mathbb{N}^{*}$, et $k$ réel $\geq 2$, une nouvelle expression de $E_{k}(x)$ sous forme de somme. Elle fournira les minorations de $E_{k}(x)$.

Nous distinguons $k \geq 3$ et $k \in[2,3$ [. Dans le premier cas nous établissons une majoration explicite de $N_{k}, N_{k} \leq M_{k}$, où $N_{k}:=\min \left\{n_{k} \in \mathbb{N}\right.$, $\left.n_{k} \geq 1 ; n \geq n_{k} \Rightarrow E_{k}(n)>0\right\}$, puis nous montrons que $E_{k}(n)$ est strictement positif pour $1 \leq n \leq M_{k}-1$. Pour cela nous préciserons les variations de la suite $\left(E_{k}(n)\right)_{1 \leq n \leq M_{k}-1}$. Pour $k \in[2,3$ [ on prouvera directement $E_{k}(n)>0$ pour tout $n \in \mathbb{N}^{*}$.

2.2. Une estimation de $E_{k}(x)$. La partie entière et la partie fractionnaire du nombre réel $x$ sont notées respectivement $\lfloor x\rfloor$ et $\{x\}$.

Lemme 1. Pour tout $u \in \mathbb{R}, u \geq 1$, pour tout $\lambda \in \mathbb{R}, \lambda \geq 1$, pour tout $x \in \mathbb{N}, x \geq 1$, pour tout $k$ réel, $k \geq 1$, on a

$$
\begin{gathered}
\sum_{n \leq u} n^{\lambda}=\frac{u^{\lambda+1}}{\lambda+1}+\left(\frac{1}{2}-\{u\}\right) u^{\lambda}+\lambda a_{\lambda}(u), \\
\left|a_{\lambda}(u)\right| \leq \frac{u^{\lambda-1}}{8}
\end{gathered}
$$




$$
\begin{aligned}
E_{k}(x)= & -\frac{x^{k+1}}{k+1} \sum_{d>x} \frac{\mu(d)}{d^{k+1}}+x^{k} \sum_{d \leq x} \frac{\mu(d)}{d^{k}}\left(\frac{1}{2}-\left\{\frac{x}{d}\right\}\right) \\
& +k \sum_{d \leq x} \mu(d) a_{k}\left(\frac{x}{d}\right)
\end{aligned}
$$

où l'on a posé

$$
a_{\lambda}(x):=\int_{0}^{x}\left(\{t\}-\frac{1}{2}\right) t^{\lambda-1} d t .
$$

Démonstration. (24) s'obtient par sommation d'Abel, (25) par application de la deuxième formule de la moyenne. (26) est une conséquence de (24) et de l'égalité (12) qui se prolonge aux $k$ réels, $k \geq 1$.

2.3. Preuve du théorème 1

Lemme 2. Pour tout $k$ réel $\geq 3$ on a

$$
N_{k} \leq M_{k}:=\left\lfloor\frac{k \zeta(k-1)}{8-4 \zeta(k)}\right\rfloor+2 .
$$

Démonstration. Soit $k$ réel $\geq 3$ et $x \in \mathbb{R}, x \geq 1$. On minore successivement chacun des termes de (26). On a

$$
-\frac{x^{k+1}}{k+1} \sum_{d>x} \frac{\mu(d)}{d^{k+1}} \geq-\frac{x}{k(k+1)}
$$

Puis, on a

$$
\sum_{d \leq x} \frac{\mu(d)}{d^{k}}\left(\frac{1}{2}-\left\{\frac{x}{d}\right\}\right) \geq \frac{1}{2}\left(1-\left(\frac{1}{2^{k}}+\ldots+\frac{1}{x^{k}}\right)\right) \geq 1-\frac{\zeta(k)}{2},
$$

donc

$$
x^{k} \sum_{d \leq x} \frac{\mu(d)}{d^{k}}\left(\frac{1}{2}-\left\{\frac{x}{d}\right\}\right) \geq x^{k}\left(1-\frac{\zeta(k}{2}\right) .
$$

$\operatorname{Par}(25)$

$$
\left|\sum_{d \leq x} \mu(d) a_{k}\left(\frac{x}{d}\right)\right| \leq \frac{x^{k-1}}{8} \sum_{d \leq x} \frac{1}{d^{k-1}}
$$

d'où

$$
k \sum_{d \leq x} \mu(d) a_{k}\left(\frac{x}{d}\right) \geq-\frac{k}{8} x^{k-1} \zeta(k-1) .
$$

On obtient ainsi

$$
E_{k}(x) \geq A_{k}(x):=\left(1-\frac{\zeta(k)}{2}\right) x^{k}-\frac{k}{8} x^{k-1} \zeta(k-1)-\frac{x}{k(k+1)} .
$$


Posons

$$
x_{k}:=\frac{k \zeta(k-1)}{8-\zeta(k)} \quad\left(\text { donc } x_{k} \geq \frac{k \zeta(k)}{8-\zeta(k)} \geq \frac{k}{4} \geq \frac{3}{4}\right) .
$$

Pour tout $z \in \mathbb{R}, z \geq 1$, nous avons

$$
\begin{aligned}
A_{k}\left(x_{k}+z\right) & =\left(1-\frac{\zeta(k)}{2}\right) z\left(x_{k}+z\right)^{k-1}-\frac{x_{k}+z}{k(k+1)} \\
& =\left(x_{k}+z\right)\left(\left(1-\frac{\zeta(k)}{2}\right) z\left(x_{k}+z\right)^{k-2}-\frac{1}{k(k+1)}\right),
\end{aligned}
$$

qui est supérieur ou égal à

$$
\left(x_{k}+z\right)\left(\left(1-\frac{\zeta(3)}{2}\right)\left(\frac{3}{4}+1\right)-\frac{1}{12}\right)>0
$$

d'où le lemme 2.

Notons que pour $k$ réel $\geq 3$ on a

$$
\frac{k \zeta(k-1)}{8-\zeta(k)} \leq \frac{2}{3}
$$

puisque $3 \zeta(k-1)-16+8 \zeta(k) \leq 3 \zeta(2)+8 \zeta(3)-16<0$.

LEMme 3. Pour tout $k$ réel et $n$ entier, $1 \leq n \leq M_{k}-2$, on a $E_{k}(n) \leq$ $E_{k}(n+1)$.

Cela achève la démonstration du théorème 1 pour $k$ réel $\geq 3$ puisque $E_{k}(1)$ est trivialement strictement positif.

Preuve du lemme 3 . Soit $k$ réel $\geq 3, n \in \mathbb{N}, 1 \leq n \leq M_{k}-2$. On a

$$
E_{k}(n+1)=E_{k}(n)+\frac{n^{k+1}}{(k+1) \zeta(k+1)}+J_{k}(n+1)-\frac{(n+1)^{k+1}}{(k+1) \zeta(k+1)} .
$$

Or

$J_{k}(n+1)-\frac{(n+1)^{k+1}}{(k+1) \zeta(k+1)}=(n+1)^{k}\left(\prod_{p \mid n+1}\left(1-\frac{1}{p^{k}}\right)-\frac{n+1}{(k+1) \zeta(k+1)}\right)$,

qui est minoré par

$$
\begin{aligned}
(n+1)^{k}\left(\frac{1}{\zeta(k)}\right. & \left.-\frac{n+1}{(k+1) \zeta(k+1)}\right) \\
& \geq \frac{(n+1)^{k}}{\zeta(k+1)}\left(\frac{\zeta(k+1)}{\zeta(k)}-\frac{(2 k / 3)+1}{k+1}\right) \\
& \geq \frac{(n+1)^{k}}{\zeta(k+1)}\left(\frac{1}{\zeta(k)}-\frac{3}{4}\right) \geq \frac{(n+1)^{k}}{\zeta(k+1)}\left(\frac{1}{\zeta(3)}-\frac{3}{4}\right)>0
\end{aligned}
$$

d'où le lemme 3. 
Démonstration du théorème 1 pour $k \in[2,3$ [. Soient $k \in$ $[2,3], x \in \mathbb{N}, x \geq 6$. (27) est encore valable; de plus,

$$
\sum_{d=1}^{x} \frac{1}{d^{k-1}} \leq \sum_{d=1}^{x} \frac{1}{d} \leq \log x+\gamma+\frac{1}{x} \leq \log x+0.778,
$$

où $\gamma$ désigne la constante d'Euler, et

$$
\sum_{d \leq x} \frac{\mu(d)}{d^{k}}\left(\frac{1}{2}-\left\{\frac{x}{d}\right\}\right) \geq 1-\frac{\zeta(k)}{2}+\frac{1}{2(x+1)^{k-1}(k-1)} .
$$

Ainsi, par (26),

$$
\begin{aligned}
E_{k}(x) \geq & \left(1-\frac{\zeta(k)}{2}\right) x^{k}-\frac{k}{8}(\log x+0.778) x^{k-1} \\
& +\frac{x^{k}}{2(x+1)^{k-1}(k-1)}-\frac{x}{k(k+1)} \\
\geq & x^{k-1}\left(\left(1-\frac{\zeta(2)}{2}\right) x-\frac{k}{8}(\log x+0.778)\right)+x\left(\frac{36}{196}-\frac{1}{6}\right) \\
= & : f_{k}(x) .
\end{aligned}
$$

La fonction

$$
g_{k}(x):=\left(1-\frac{\zeta(2)}{2}\right) x-\frac{k}{8}(\log x+0.778)
$$

est strictement croissante sur $\left[6,+\infty\left[\right.\right.$, avec $g_{k}(x) \geq g_{3}(6)>0$; ceci assure $E_{k}(n)>0$ pour tout $n$ entier $\geq 6$.

Toujours pour $k \in[2,3], E_{k}(x)$ est trivialement $>0$ pour $x \in\{1,2,3\}$. D'autre part, pour $x=4$ on a l'équivalence

$$
E_{k}(4)>0 \Leftrightarrow 3^{k}+4^{k}-1>\frac{4^{k+1}}{(k+1) \zeta(k+1)} .
$$

Les deux membres de cette dernière inégalité sont des fonctions croissantes de $k$ (noter la croissance de $k \mapsto 4^{k+1} /(k+1)$ ) et on vérifie les cinq inégalités $3^{2+0.2 n}+4^{2+0.2 n}-1>\frac{4^{3+0.2(n+1)}}{(3+0.2(n+1)) \zeta(3+0.2(n+1))}, \quad n \in\{0,1,2,3,4\}$.

Le raisonnement est le même pour $x=5$ :

$$
E_{k}(5)>0 \Leftrightarrow 3^{k}+4^{k}+5^{k}-2>\frac{5^{k+1}}{(k+1) \zeta(k+1)}
$$

et l'on vérifie

$$
3^{2+0.2 n}+4^{2+0.2 n}+5^{2+0.2 n}-2>\frac{5^{3+0.2(n+1)}}{(3+0.2(n+1)) \zeta(3+0.2(n+1))},
$$

$n \in\{0,1,2,3,4\}$, ce qui achève la démonstration du théorème 1 . 
2.4. Principe de la démonstration du théorème 2. Nous avons, par définition,

$$
E_{k}\left(n^{-}\right)=\sum_{m<n} J_{k}(m)-\frac{n^{k+1}}{(k+1) \zeta(k+1)} \quad\left(k \text { réel }>1, n \in \mathbb{N}^{*}\right) .
$$

La démonstration du théorème 2 est similaire à celle du théorème 1 : on distingue encore $k \geq 3$ et $k \in[2,3$ [. Pour $k \geq 3$ on calcule explicitement, avec (26), un entier $L_{k}$ tel que $n \in \mathbb{N}, n \geq L_{k} \Rightarrow E_{k}\left(n^{-}\right)<0$, notamment,

$$
L_{k}=\left\lfloor\frac{k}{8} \cdot \frac{\zeta(k-1) \zeta(k)}{1-\zeta(k)^{2} / 2}\right\rfloor+2 ;
$$

puis on établit que la suite $\left(E_{k}\left(n^{-}\right)\right)_{1 \leq n \leq L_{k}-1}$ est strictement décroissante. Pour $k \in\left[2,3\right.$ [ on prouve plus directement $E_{k}\left(n^{-}\right)<0$ pour tout $n \in \mathbb{N}^{*}$ en utilisant des résultats, notamment numériques, de [4].

3. Comportement de $s_{k}$ au voisinage à droite de $k=1$

3.1. Une preuve de $\lim _{k \rightarrow 1, k>1} s_{k}=+\infty$. On a l'analogue du théorème 3.3 de [1] pour $k=1$ :

$$
E_{1}(n)=n \sum_{d=1}^{+\infty} \frac{\mu(d)}{d}\left(\frac{1}{2}-\left\{\frac{n}{d}\right\}\right)+O(n)
$$

qui entraîne

$$
E_{1}(n)=-n \sum_{d=1}^{+\infty} \frac{\mu(d)}{d}\left\{\frac{n}{d}\right\}+O(n) .
$$

D'autre part, pour tout $k$ réel $>1$,

$$
\sum_{d=1}^{+\infty} \frac{\mu(d)}{d^{k}}\left(\frac{1}{2}-\left\{\frac{n}{d}\right\}\right)=\frac{1}{2 \zeta(k)}-n \sum_{d=n+1}^{+\infty} \frac{\mu(d)}{d^{k+1}}-\sum_{d=1}^{n} \frac{\mu(d)}{d^{k}}\left\{\frac{n}{d}\right\}
$$

et

$$
\left|\frac{1}{2 \zeta(k)}-n \sum_{d=n+1}^{+\infty} \frac{\mu(d)}{d^{k+1}}\right| \leq \frac{3}{2} .
$$

Soit $M$ réel $>0$. Puisque $E_{1}(n)=\Omega_{+}\left(n \log _{4} n\right)$, (30) entraîne qu'il existe $m_{M}$, entier $>0$, tel que

$$
-\sum_{d=1}^{m_{M}} \frac{\mu(d)}{d}\left\{\frac{m_{M}}{d}\right\}>M .
$$

Par suite, comme

$$
\lim _{\substack{k \rightarrow 1 \\ k>1}}-\sum_{d=1}^{m_{M}} \frac{\mu(d)}{d^{k}}\left\{\frac{m_{M}}{d}\right\}=-\sum_{d=1}^{m_{M}} \frac{\mu(d)}{d}\left\{\frac{m_{M}}{d}\right\},
$$


il existe $k_{M}$, réel $>1$, tel que

$$
1<k<k_{M} \Rightarrow-\sum_{d=1}^{m_{M}} \frac{\mu(d)}{d^{k}}\left\{\frac{m_{M}}{d}\right\}>M .
$$

Ainsi, avec (31) et (32),

$$
1<k<k_{M} \Rightarrow \sum_{d=1}^{m_{M}} \frac{\mu(d)}{d^{k}}\left(\frac{1}{2}-\left\{\frac{m_{M}}{d}\right\}\right)>M-\frac{3}{2} .
$$

Le lemme 1 de [4] étant encore valable, il suit que $s_{k}>M-3 / 2$ pour $1<k<k_{M}$. D'où le résultat annoncé.

Conséquences : 1) Avec (19):

$$
\lim _{\substack{k \rightarrow 1 \\ k>1}} I_{k}=-\infty
$$

2) Avec (33) : le théorème $\mathrm{C}$ n'admet pas de prolongement au voisinage de 1 (et a fortiori le théorème 1 non plus).

3) Toujours avec (19) : le théorème 2 n'admet pas de prolongement au voisinage de 1 : pour $k>1$ suffisamment proche de $1, E_{k}\left(n^{-}\right)$est strictement positif pour une infinité de valeurs de l'entier $n$.

3.2. Esquisse de la preuve du théorème 3. Nous employons la méthode des moyennes sur des progressions arithmétiques. Nous posons, pour $x \in \mathbb{R}^{+}$ et $k$ réel $>1$,

$$
\begin{aligned}
R_{k}(x) & :=\sum_{n \leq x} \frac{J_{k}(n)}{n^{k}}-\frac{x}{\zeta(k+1)}, \\
h_{k}(x) & :=\sum_{d=1}^{+\infty} \frac{\mu(d)}{d^{k}}\left(\frac{1}{2}-\left\{\frac{x}{d}\right\}\right) .
\end{aligned}
$$

Nous définissons $s$ par

$$
\left\{\begin{array}{l}
s(0)=0, s(x):=1 / 2-x \text { pour } 0<x<1, \\
s \text { périodique de période } 1 .
\end{array}\right.
$$

En adaptant les démonstrations de [3] nous avons obtenu :

Lemme 4. Pour $q \in \mathbb{N}^{*}, N \in \mathbb{N}^{*}, q \leq \exp \left(c_{2} \sqrt{\log N}\right)$, et $\alpha \in \mathbb{R} \backslash \mathbb{N}$, $0<\alpha<q$,

$$
\sum_{n=1}^{N} R_{k}(n q+\alpha)=C_{k}(q, \alpha) N-\frac{N}{2 \zeta(k)}+O\left(N \exp \left(-c_{2} \sqrt{\log N}\right)\right),
$$

où $c_{2}$ est une constante strictement positive et

$$
C_{k}(q, \alpha):=\frac{1}{\zeta(k+1)} \prod_{p \mid q}\left(1-p^{-k-1}\right)^{-1} \sum_{d \mid q} \mu(d) \frac{s(\alpha / d)}{d^{k}} .
$$


Lemme 5. Pour tout $x \in \mathbb{R}^{+}$on a

$$
R_{k}(x)=h_{k}(x)-\frac{1}{2 \zeta(k)} .
$$

Preuve du résultat annoncé. Soit $k$ réel $>1$, la relation (13) étant en fait valable pour tout $k$ réel $>1$, on a

$$
s_{k}=\limsup _{x \rightarrow+\infty} h_{k}(x) .
$$

Soit $z$ réel $>0$ vérifiant $\operatorname{card}\{p \leq z: p$ premier, $p \equiv 3(\bmod p)\}$ pair, et $q_{z}=\prod_{p \leq z, p \equiv 3(\bmod 4)} p ; q_{z}$ est congru à 1 modulo 4 et, facilement,

$$
C_{k}\left(q_{z}, \frac{q_{z}}{4}\right)=\frac{1}{4 \zeta(k+1)}\left(\prod_{p \mid q_{z}}\left(1-p^{-k-1}\right)^{-1}\right) \sum_{d \mid q_{z}} \frac{1}{d^{k}} .
$$

Par le lemme 4 (en choisissant $\alpha=q_{z} / 4$ ) et par le lemme 5 , on obtient, en faisant tendre $N$, puis $z$, vers l'infini,

$$
s_{k} \geq \frac{1}{5 \zeta(k+1)}\left(\prod_{p \equiv 3(\bmod 4)}\left(1-p^{-k-1}\right)^{-1}\right) \prod_{p \equiv 3(\bmod 4)}\left(1+\frac{1}{p^{k}}\right),
$$

ce qui prouve que l'on peut choisir $c$ inférieure à

$$
\begin{aligned}
\inf _{K>1} & \frac{1}{5 \zeta(K+1)}\left(\prod_{p \equiv 3(\bmod 4)}\left(1-p^{-K-1}\right)^{-1}\right) \\
& \times \sqrt{\prod_{p \equiv 3(\bmod 4)}\left(1-p^{-2 K}\right)} \sqrt{\left(\prod_{p \equiv 1(\bmod 4)}\left(1-p^{-K}\right)\right) \prod_{p \equiv 3(\bmod 4)}\left(1+p^{-K}\right)} \\
& \geq \inf _{K>1} \frac{1}{5 \zeta(K+1)} \sqrt{\left(\prod_{p \equiv 1(\bmod 4)}\left(1-p^{-K}\right)\right) \prod_{p \equiv 3(\bmod 4)}\left(1+p^{-K}\right)} .
\end{aligned}
$$

Nous avons

$$
\begin{aligned}
& \left(\prod_{p \equiv 1(\bmod 4)}\left(1-p^{-k}\right)\right) \prod_{p \equiv 3(\bmod 4)}\left(1+p^{-k}\right) \\
& \quad \geq \exp \left(-\sum_{p \equiv 1(\bmod 4)}\left(p^{-k}+p^{-2 k}\right)+\sum_{p \equiv 3(\bmod 4)}\left(p^{-k}-p^{-2 k}\right)\right) \\
& \quad \geq \exp \left(-\sum_{p \geq 3} p^{-2}+\sum_{p \equiv 3(\bmod 4)} p^{-k}-\sum_{p \equiv 1(\bmod 4)} p^{-k}\right) .
\end{aligned}
$$

Reste à minorer $\sum_{p \equiv 3(\bmod 4)} p^{-k}-\sum_{p \equiv 1(\bmod 4)} p^{-k}$. 
Soient $\chi_{0}$ et $\chi_{1}$ les deux caractères modulo $4, \chi_{0}$ étant le caractère principal. $\chi_{1}$ est défini par

$$
\chi_{1}(n)= \begin{cases}-1 & \text { si } n \equiv 3(\bmod 4) \\ 1 & \text { si } n \equiv 1(\bmod 4) \\ 0 & \text { si } n \equiv 0(\bmod 2)\end{cases}
$$

Pour $l \in\{1,3\}$ on a

$$
\sum_{\nu=1}^{+\infty} \sum_{p^{\nu} \equiv l(\bmod 4)} \nu^{-1} p^{-\nu k}=\frac{1}{\varphi(4)} \sum_{\chi} \bar{\chi}(l) \log L(k, \chi),
$$

d'où

$$
\begin{aligned}
& \sum_{p \equiv 3(\bmod 4)} p^{-k}-\sum_{p \equiv 1(\bmod 4)} p^{-k} \\
& =-\log L\left(k, \chi_{1}\right)+\sum_{\nu=2}^{+\infty}\left(\sum_{p^{\nu} \equiv 1(\bmod 4)} \nu^{-1} p^{-\nu k}-\sum_{p^{\nu} \equiv 3(\bmod 4)} \nu^{-1} p^{-\nu k}\right) .
\end{aligned}
$$

Par définition

$$
L\left(k, \chi_{1}\right)=\sum_{n=1}^{+\infty} \frac{\chi_{1}(n)}{n^{k}}
$$

donc $0<L\left(k, \chi_{1}\right)<1$ (cf. l'expression de $\chi_{1}(n): L\left(k, \chi_{1}\right)$ est une série alternée). Ainsi

$$
\begin{aligned}
\sum_{p \equiv 3(\bmod 4)} p^{-k}- & \sum_{p \equiv 1(\bmod 4)} p^{-k} \\
& =\sum_{\nu=2}^{+\infty}\left(\sum_{p^{\nu} \equiv l(\bmod 4)} \nu^{-1} p^{-\nu k}-\sum_{p^{\nu} \equiv 3(\bmod 4)} \nu^{-1} p^{-\nu k}\right) \\
& \geq-\sum_{\nu=2}^{+\infty} \sum_{p^{\nu} \equiv 3(\bmod 4)} \nu^{-1} p^{-\nu k} \geq-\sum_{\nu \geq 2, p \equiv 3(\bmod 4)} \frac{1}{2 p^{\nu k}} \\
& \geq-\sum_{p \equiv 3(\bmod 4)} \frac{1}{2 p(p-1)} .
\end{aligned}
$$

Cela achève la démonstration du théorème 3 .

4. Preuve du théorème 4. Pour montrer la croissance de la suite $\left(I_{k}\right)_{k \geq 2}$, Pétermann s'est appuyé sur un encadrement de $I_{k}$. Notre démonstration repose sur une estimation exacte de $I_{k}$. Nous utiliserons à nouveau des résultats de [4].

Lemme 6. Pour tout $k>1$ on a $s_{k}=\sup _{n \in \mathbb{N}} h_{k}(n)$. 
Lemme 7. $s_{k}$ est dérivable à droite sur $] 1,+\infty[$.

Re marque. $s_{k}$ et $I_{k}$ sont donc continues sur cet intervalle et $I_{k}$ y est dérivable à droite.

LeMme 8. Si $D(k):=\sum_{d=1}^{+\infty} c_{d} / d^{k} \leq 0$ pour $k=K$ et si $c_{d} \leq 0$ pour $d<\Delta$, et $c_{d} \geq 0$ pour $d \geq \Delta$, alors $D(k) \leq 0$ pour tout $k \geq K$.

Le lemme 6 est une conséquence immédiate de la démonstration de [4](2.4) $: s_{k}=\sup _{n \in \mathbb{Z}} h_{k}(n)$; celle-ci est, en fait, valable pour tout $k$ réel $>1$.

Le lemme 8 est une légère variante du lemme 3 de [4].

Preuve du lemme 7. Nous nous inspirons du travail de Pétermann. Pour $n$ entier $\geq 1$ nous notons $p_{n}$ le $n$-ième nombre premier; pour $r$ entier $\geq 1$ nous posons $P_{r}:=\prod_{p \leq p_{r}} p$ et, pour $k$ réel $>1$,

$$
\eta_{0}(k):=-\sum_{d=1}^{+\infty} \frac{|\mu(d)|}{2 d^{k}}+\sum_{\mu(d)=1} \frac{1}{d^{k+1}} .
$$

Pour $r \geq 1$, les différentes classes de congruence $m\left(P_{r}\right)(m=0,1, \ldots$ $\left.\ldots, P_{r}-1\right)$ correspondent chacune à un système de congruences $S_{r, m}:=$ $\left\{m_{d}(d): d \mid P_{r}\right\}$ (où par convention $\left.0 \leq m_{d}-1\right)$. Si $n \equiv m\left(\bmod P_{r}\right)$ alors, pour tout $k$ réel $>1$,

$$
h_{k}(n)=\sum_{d \mid P_{r}} \frac{\mu(d)}{d^{k}}\left(\frac{1}{2}-\frac{m_{d}}{d}\right)+\sum_{d \nmid P_{r}} \frac{\mu(d)}{d^{k}}\left(\frac{1}{2}-\left\{\frac{n}{d}\right\}\right)
$$

et l'on a

$$
h_{k}(n)=\eta_{0}(k)+\varepsilon_{r, m}(k)+\delta_{r, n}(k)
$$

où

$$
\varepsilon_{r, m}(k):=\sum_{d \mid P_{r}} \frac{a_{d}}{d^{k+1}}
$$

avec

$$
a_{d}=a_{d, r}(m):= \begin{cases}m_{d} & \text { si } \mu(d)=-1 \\ d-1-m_{d} & \text { si } \mu(d)=1\end{cases}
$$

et

$$
\begin{aligned}
0<\delta_{r, n}(k) & :=\sum_{\substack{d \nmid P_{r} \\
\mu(d)=-1}} \frac{1}{d^{k}}\left\{\frac{n}{d}\right\}+\sum_{\substack{d \nmid P_{r} \\
\mu(d)=-1}} \frac{1}{d^{k}}\left(1-\frac{1}{d}-\left\{\frac{n}{d}\right\}\right) \\
& <\frac{1}{(k-1) p_{r}^{k-1}}
\end{aligned}
$$


Soient

$$
\varepsilon_{r_{M}}(k):=\sup _{0 \leq m \leq P_{r}-1} \varepsilon_{r, m}(k) \text { et } \varepsilon_{M}(k):=\lim _{r \rightarrow+\infty} \varepsilon_{r_{M}}(k)
$$

(qui existe dans $\mathbb{N}$ puisque la suite $\left(\varepsilon_{r_{M}}(k)\right)_{r \in \mathbb{N}^{*}}$ est croissante et majorée $\operatorname{par} \zeta(k) / \zeta(2 k)-\zeta(k+1) / \zeta(2 k+2))$.

Par (34), (35) et le lemme 6 nous avons $s_{k}=\eta_{0}(k)+\varepsilon_{M}(k)$, ce qui équivaut à

$$
s_{k}=-\frac{\zeta(k)}{2 \zeta(2 k)}+\frac{\zeta(k+1)}{2 \zeta(2 k+2)}+\frac{1}{2 \zeta(k+1)}+\varepsilon_{M}(k) .
$$

Or, pour $r \geq 1$ et $m \in\left\{0,1, \ldots, P_{r}-1\right\}, k \mapsto \varepsilon_{r, m}(k)$ étant convexe, il en est de même de $k \mapsto \varepsilon_{M}(k)$. D'où, avec (36), le lemme 7 .

Soit $K>1$ tel que $s_{K} \geq 1 / 2$. Pour tout $n \in \mathbb{N}$ on a, par le lemme 6 ,

$$
h_{K}(n) \leq s_{K}=\left(s_{K}+\frac{\beta}{2^{K}}\right)-\frac{1}{2} \sum_{d=3}^{+\infty} \frac{|\mu(d)|}{d^{K}}
$$

où

$$
\beta:=2^{K} \frac{1}{2} \sum_{d=3}^{+\infty} \frac{|\mu(d)|}{d^{K}} \quad(\beta=\beta(K)) .
$$

Par conséquent, par le lemme 8,

$$
k \geq K \Rightarrow h_{k}(n) \leq\left(s_{K}+\frac{\beta}{2^{k}}\right)-\frac{1}{2} \sum_{d=3}^{+\infty} \frac{|\mu(d)|}{d^{k}}
$$

d'où

$$
k \geq K \Rightarrow s_{k} \leq\left(s_{K}+\frac{\beta}{2^{k}}\right)-\frac{1}{2} \sum_{d=3}^{+\infty} \frac{|\mu(d)|}{d^{k}} .
$$

Il suit de cela que la dérivée à droite de $s_{k}$ en $K$ est majorée par

$$
-\frac{\beta}{2^{K}} \log 2+\frac{1}{2} \sum_{d=3}^{+\infty} \frac{|\mu(d)|}{d^{K}} \log d .
$$

Ceci équivaut à

$$
0 \leq I_{K_{d}}^{\prime}+\frac{\zeta^{\prime}(K+1)}{\zeta(K+1)^{2}}-\frac{\log 2}{2} \sum_{d=3}^{+\infty} \frac{|\mu(d)|}{d^{K}} \log d
$$

où $I_{K_{d}}^{\prime}$ désigne la dérivée à droite de $I_{k}$ au point $K$. Posons

$$
F(K):=\frac{\zeta^{\prime}(K+1)}{\zeta(K+1)^{2}}-\frac{\log 2}{2} \sum_{d=3}^{+\infty} \frac{|\mu(d)|}{d^{K}} \log d .
$$

Avec la calculette du logiciel de calcul arithmétique PARI, nous avons obtenu $F(1.961 \ldots)=0$. Nous allons établir $F(K)<0$ pour tout $K \geq 1.97$. 
Nous commençons par prouver cette inégalité pour $K \geq 3$. Pour tout $K>1$, $F(K)$ est majoré par

$$
\begin{aligned}
\left(-\frac{\log 2}{2^{K+1}}\right. & \left.-\frac{\log 3}{3^{K+1}}-\frac{\log 5}{5^{K+1}}+\sum_{d=6}^{+\infty} \frac{|\mu(d)|}{d^{K+1}} \log d\right) \\
& -\frac{\log 2}{2}\left(\frac{1}{3^{K}}+\frac{1}{5^{K}}+\sum_{d=6}^{+\infty} \frac{|\mu(d)|}{d^{K}}\right) \\
& +\frac{1}{2}\left(\frac{\log 3}{3^{K}}+\frac{\log 5}{5^{K}}+\left(\log 5+\frac{1}{K-1}\right) \frac{1}{(K-1) 5^{K-1}}\right) \\
\leq & \left(-\frac{\log 2}{2^{K+1}}+\frac{1}{2}\left(\log 5+\frac{1}{K-1}\right) \frac{1}{K-1} \cdot \frac{1}{5^{K-1}}\right) \\
& +\left(\frac{\log (3 / 8)}{2 \cdot 3^{K+1}}+\frac{\log (125 / 32)}{2 \cdot 5^{K+1}}\right)+\sum_{d=6}^{+\infty} \frac{|\mu(d)|}{d^{K}}\left(\mu(d) \frac{\log d}{d}-\frac{\log 2}{2}\right)
\end{aligned}
$$

qui est $<0$ pour $K \geq 3$.

Nous écrivons maintenant $F(K)$ sous forme d'une somme de deux séries de Dirichlet, l'une à coefficients positifs, l'autre à coefficients négatifs : $F(K)=f(K)+g(K)$ où

$$
\begin{aligned}
& f(K):=\sum_{d=5}^{+\infty} \frac{|\mu(d)|}{d^{K}}\left(\mu(d) \frac{\log d}{d}-\frac{\log 2}{2}+\frac{\log d}{2}\right), \\
& g(K):=-\frac{\log 2}{2^{K+1}}+\frac{1}{3^{K}}\left(\frac{\log 3}{6}-\frac{\log 2}{2}\right) .
\end{aligned}
$$

$f$ et $g$ sont respectivement croissantes et décroissantes sur $] 1,+\infty[;$ un calcul machine similaire à celui effectué à la fin de la démonstration du théorème 1 , avec cette fois des pas de $1 / 80$, permet d'aboutir à la majoration de $F(K)$ recherchée pour $1.97 \leq K \leq 3$.

Pour terminer la démonstration du théorème 4 , il reste à verifier $s_{k} \geq 1 / 2$ pour tout $k \geq 1.97$. Nous montrons que cette inégalité est vraie pour tout $k>1$.

Le point (2.2) de [4] s'étend aux $k$ réels $>1$ :

$$
s_{k} \geq h_{k}(-1)=\frac{1}{\zeta(k+1)}-\frac{1}{2 \zeta(k)} \geq \frac{1}{2}+\frac{1}{2 \cdot 3^{k+1}}-\frac{1}{2(k-1) 5^{k-1}},
$$

qui est $>1 / 2$ pour $k \geq 3$. Les fonctions $k \mapsto 1 / \zeta(k+1)$ et $k \mapsto 1 /(2 \zeta(k))$ sont croissantes sur $] 1,+\infty[$. Nous avons vérifié que

$$
\frac{1}{\zeta\left(2+\frac{n-1}{1000}\right)}-\frac{1}{2 \zeta\left(1+\frac{n}{1000}\right)} \geq \frac{1}{2} \quad \text { pour tout } n \in \mathbb{N}, 1 \leq n \leq 2000,
$$

ce qui achève la démonstration de ce théorème. 
Nous conjecturons que $I_{k}$ est strictement croissante sur $] 1,+\infty[$.

L'auteur remercie vivement Y.-F. S. Pétermann pour les remarques et commentaires qu'il a apporté à ce travail.

\section{Bibliographie}

[1] S. D. Adhikari and A. Sankaranarayanan, On an error term related to the totient function $J_{k}(n)$, J. Number Theory 34 (1990), 177-188.

[2] P. Erdös and H. N. Shapiro, On the changes of sign of a certain error function, Canad. J. Math. 3 (1951), no. 3, 375-384, et no. 4, 385.

[3] H. L. Montgomery, Fluctuations in the mean of Euler's phi function, Proc. Indian Acad. Sci. Math. Sci. 97 (1987), 239-245.

[4] Y.-F. S. Pétermann, Oscillations d'un terme d'erreur lié à la fonction totient de Jordan, Sém. Théor. Nombres Bordeaux 3 (1991), 311-335.

[5] J. J. Sylvester, Collected Papers IV, Cambridge, 1912, 101-109.

34, rue Limogeanne

24000 Périgueux, France 